\title{
Three-dimensionally aligned sulfur electrodes by directional freeze tape casting
}

Yoon Hwa, ${ }^{a, b,+\vec{t}}$ Eongyu Yi, ${ }^{a,+}$ Hao Shen, ${ }^{c, d}$ Younghoon Sung, ${ }^{b}$ Jiawei Kou, ${ }^{d}$ Kai Chen, ${ }^{d}$ Dilworth Y. Parkinson, ${ }^{c}$ Marca M. Doeffa, ${ }^{a}$ and Elton J. Cairns ${ }^{a, b, *}$

a Energy Storage and Distributed Resources Division, Lawrence Berkeley National Laboratory, Berkeley, California, 94720, USA

b Department of Chemical and Biomolecular Engineering, University of California, Berkeley, California, 94720, USA

c Advanced Light Source, Lawrence Berkeley National Laboratory, Berkeley, California, 94720, USA

d Center for Advancing Materials Performance from the Nanoscale (CAMP-Nano), State Key Laboratory for Mechanical Behavior of Materials, Xi'an Jiaotong University, Xi'an, Shaanxi 710049, China

\section{Corresponding Author}

* Elton J. Cairns, ejcairns@1bl.gov

* Marca M. Doeff, mmdoeff@lbl.gov 


\section{Supplemental Experimental methods}

Synthesis of CTAB modified S-GO composite (S-GO-CTA): The S-GO-CTA composite was prepared via our published method ${ }^{1,2}$. Briefly, $580 \mathrm{mg}$ of $\mathrm{Na}_{2} \mathrm{~S}$ powder (Alfa Aesar, $\mathrm{Na}_{2} \mathrm{~S}$, anhydrous) was dissolved in $25 \mathrm{~mL}$ of ultrapure water and then $720 \mathrm{mg}$ of S powder (Alfa Aesar, S, $\sim 325$ mesh, 99.5\%) was added to the $\mathrm{Na}_{2} \mathrm{~S}$ solution and magnetically stirred at $60{ }^{\circ} \mathrm{C}$ until the solution became transparent orange. $2.5 \mathrm{mM}(164 \mathrm{mg})$ of $\mathrm{CH}_{3}\left(\mathrm{CH}_{2}\right)_{15} \mathrm{~N}(\mathrm{Br})\left(\mathrm{CH}_{3}\right)_{3}(\mathrm{CTAB}$, Sigma Aldrich) was added into the diluted GO suspension (ACS materials, $180 \mathrm{mg}$ of GO in $180 \mathrm{~mL}$ of ultrapure water) and magnetically stirred for $2 \mathrm{~h}$. Then the prepared $\mathrm{Na}_{2} \mathrm{~S}_{\mathrm{x}}$ solution was added to the GO-CTAB mixture and stirred overnight. The as-prepared $\mathrm{Na}_{2} \mathrm{~S}_{\mathrm{x}}-\mathrm{GO}-\mathrm{CTAB}$ mixture was slowly added to $100 \mathrm{~mL}$ of $2.0 \mathrm{M}$ formic acid ( $\mathrm{HCOOH}$, Aqua Solutions) and stirred for $2 \mathrm{~h}$ to precipitate elemental $\mathrm{S}$ onto the GO. To remove salts and impurities from the $\mathrm{S}-\mathrm{GO}-\mathrm{CTA}$ suspension, the supernatant was discarded followed by adding ultrapure water. The process was repeated until the suspension reached a $\mathrm{pH}$ of 7.0. Finally, the $\mathrm{S}-\mathrm{GO}-\mathrm{CTA}$ composite in approximately $100.0 \mathrm{~mL}$ of water was frozen at $-85.0{ }^{\circ} \mathrm{C}$ and lyophilized (Labconco) at $0.1 \mathrm{mbar}$. The $\mathrm{S}-\mathrm{GO}-\mathrm{CTA}$ composite was heat-treated at $155^{\circ} \mathrm{C}$ for $18 \mathrm{~h}$ in an $\mathrm{Ar}$ atmosphere.

Electrode fabrication: The $\mathrm{S}-\mathrm{GO}-\mathrm{CTA}$ electrodes were prepared using a slurry consisting of S-GO-CTA, conductive carbon (Ketjen Black:CNT=97:3, w/w), and SBR:CMC binder $(1: 1, w / w)$ at a weight ratio of 80:10:10 in ultrapure water. A research scale freeze tape-caster (GLA-RC2, Glacigen) was used to prepare porous electrodes with aligned pore structures. The prepared slurries were cast onto $\mathrm{Al}$ foil using a reservoir type doctor blade. The foil was pulled at a rate of 3-4 mm min ${ }^{-1}$ over the freezing bed held at $-7^{\circ} \mathrm{C}$. The slurry froze as it entered the freezing zone and the pulling continued until the entire cast was frozen. Subsequently, the ice was sublimed by freeze 
drying the samples (FreeZone Freeze Dryer, Labconco). S-GO-CTA electrodes were also prepared using a conventional tape casting method to serve as a control. For the catholyte cell tests, three different GO electrodes were prepared: (1) freeze tape cast GO electrodes in which the slurry was prepared by mixing a single layered GO suspension in water and a SBR suspension in water; (2) tape cast GO electrode in which the slurry was prepared by adding freeze-dried GO powder into the SBR suspension in water; (3) dense GO film electrode: tape cast GO electrode in which the slurry was prepared by mixing a single layered GO suspension in water and a SBR suspension in water. The weight ratio between GO and SBR was 8:2. Freeze tape casting was performed under conditions similar to those described above. The slurries were cast onto Al foil using a conventional tape casting method and dried at room temperature for $6.0 \mathrm{~h}$, then dried in a vacuum oven at $50.0^{\circ} \mathrm{C}$ overnight to remove any residual water.

Electrochemical tests: An electrolyte composed of 1.0 M LiTFSI and 0.1 $\mathrm{M} \mathrm{LiNO}_{3}$ in DOL/DME $(1: 1, v / v)$ was used. Coin cells (2032-type) were fabricated with a Li metal foil as counter/reference electrode, $30 \mu \mathrm{L}$ of the electrolyte, and a porous polypropylene as a separator (Celgard 2400), in an Ar glovebox. Galvanostatic cycling tests of the coin cells were performed using a battery cycler (Maccor) at selected current densities. The electrochemical impedance in the charged state was measured from $10 \mathrm{mHz}$ to $1 \mathrm{MHz}$ using a potentiostat equipped with a frequency response analyzer (Biologic VSP). Cyclic voltammetry was performed at a scan rate of $0.1 \mathrm{mV} / \mathrm{s}$. For the catholyte cell test, the prepared GO electrodes were tested with a catholyte composed of DOL:DME $(1: 1, v / v)$ with $1 \mathrm{M}$ LiTFSI, $0.1 \mathrm{MLiNO}_{3}$ and $0.33 \mathrm{M} \mathrm{Li}_{2} \mathrm{~S}_{6}\left(2 \mathrm{mgS} / \mathrm{cm}^{2}\right.$ per GO electrode was in the electrolyte). 
Material characterization: The morphologies of the S-GO-CTA electrodes, the GO electrodes and the cycled GO electrodes in the catholyte cells were observed using a scanning electron microscope (SEM, ZEISS Gemini Ultra 55). Thermogravimetric analysis (TGA, TA Instruments Q5000) was used to determine the $\mathrm{S}$ content in the $\mathrm{S}-\mathrm{GO}-\mathrm{CTA}$ composites by heating the sample to $600{ }^{\circ} \mathrm{C}$ in a $\mathrm{N}_{2}$ atmosphere at $5{ }^{\circ} \mathrm{C} / \mathrm{min}$.

Polysulfide adsorption test: For the polysulfide adsorption test of the $\mathrm{NGO}, 3,9$ or $15 \mathrm{mg}$ of the conventionally-dried GO powder or the freeze-dried GO powder was put into a test solution composed of $1.25 \mathrm{mM} \mathrm{Li}_{2} \mathrm{~S}_{8}$ in $5 \mathrm{~mL}$ of DOL/DME mixture $(1: 1, v / v)$ overnight. For absorbance measurements (Shimadzu UV-3600 Plus), polysulfide solutions were collected after the test, then diluted to $1 \%$ by mixing in DOL/DME solutions (test solution:mixture solution $=1: 99, \mathrm{v} / \mathrm{v}$ ). The diluted test solution was added to a quartz cuvette, sealed and quickly transferred to the instrument for measurement.

Micro X-ray computed tomography (micro X-ray CT): The synchrotron micro X-ray CT experiment was performed on Beamline 8.3.2 at the Advanced Light Source (ALS), which offers the opportunity to reconstruct the structure of the freeze tape cast electrode with high resolution to 1 $\mu \mathrm{m}$. All electrodes were punched to $3 \mathrm{~mm}$ diameter discs. The samples were scanned with a $24 \mathrm{keV}$ monochromatic X-ray beam at a resolution of $\sim 1 \mu \mathrm{m}$. The raw data were analyzed by ImageJ and the 3D model was reconstructed using Avizo software. For a better view of the structure, a subvolumes with the size of $490 \times 620 \times 120 \mu \mathrm{m}$ for the FTC-200 and 300 electrodes, and $500 \times$ $400 \times 100 \mu \mathrm{m}$ for FTC-GO electrode are displayed. Because of the high periodicity in the distribution of layers, we were able to transfer the patterns through fast Fourier transform (FFT). Several symmetrical peaks which correspond to the interlayer spacing are shown on the FFT pattern. 
The distance between two relevant peaks is inversely proportional to the interlayer spacing. To strengthen the peak intensity in the FFT pattern, several conventional steps for image processing were adopted to guarantee that the peak search goes smoothly. First, we use the log transformation which can be expressed as the equation to recalculate the FFT pattern which serves to decrease the large overall fluctuation of the signal in the FFT pattern.

$$
I_{\text {new }}=\lg \left(1+I_{\text {origin }}\right)
$$

Here, $\mathrm{I}_{\text {origin }}$ is the original intensity of the signals in the FFT pattern, and $\mathrm{I}_{\text {new }}$ refers to the calculated value. The background signal like concentric rings which come from the randomly distributed particles or noise in the raw pattern are removed then. Signals with intensity lower than a critical value are considered as noise and set to zero. In this step, the critical value is set as 1.15 times the average intensity of all pixels empirically. Also, the median filter is used to remove the pepper \& salt noise and the center peak is blocked. After all these steps, we are able to search for the peak in the FFT pattern, and the peak center can be located by 2D Gaussian fitting. We pick peaks which have an integrated intensity higher than 10 which is set empirically here as valid peaks to calculate the interlayer spacing after calculating the integrated intensity. High peak intensity in the FFT pattern refers to the high periodicity in the raw pattern. So, the main periodicities, or the main interlayer spacing is measured frame by frame, and the average interlayer spacing is calculated per frame if several periodicities exist in one FFT pattern.

\section{Supplemental Figures}




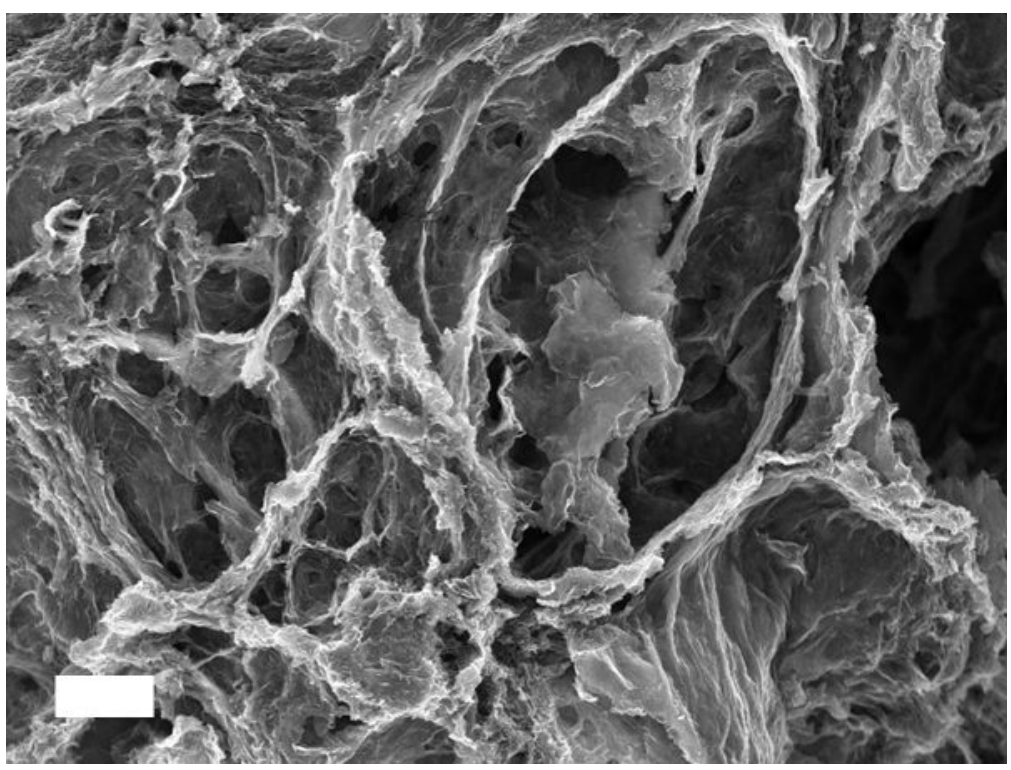

Figure S1 SEM image of the S-GO-CTA composite (scale bar: $2 \mu \mathrm{m}$ ).

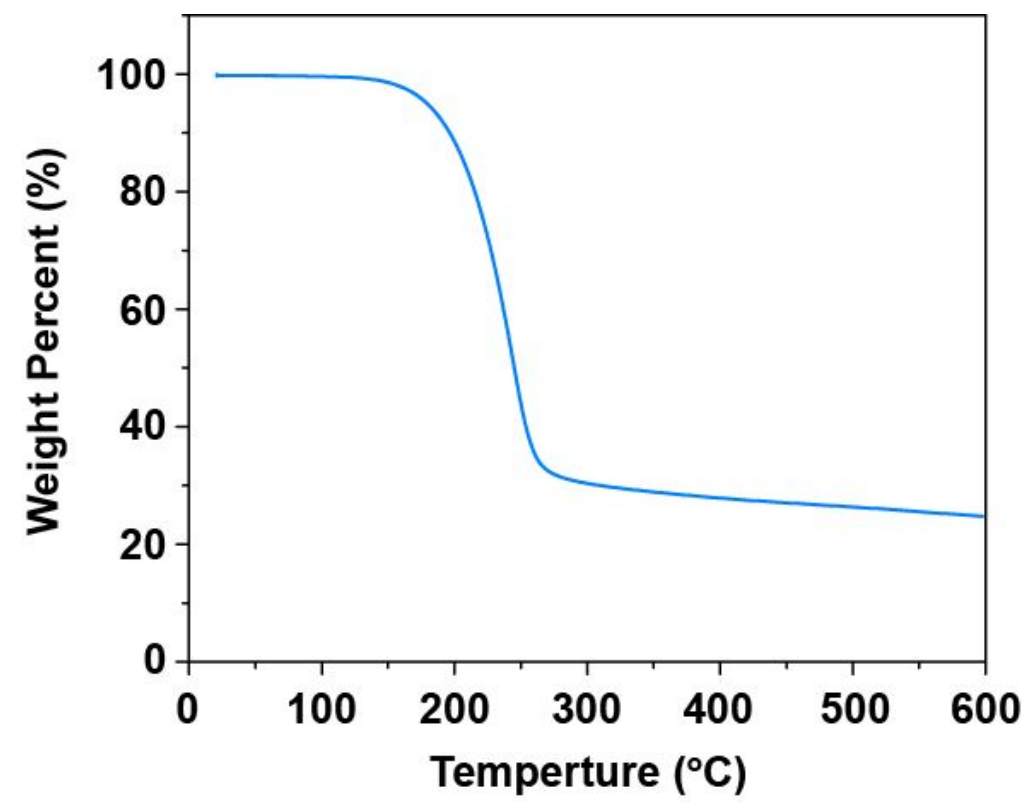

Figure S2 TGA result of the S-GO-CTA composite under Ar atmosphere with a temperature ramping rate of $5.0^{\circ} \mathrm{C} / \mathrm{min}$. 


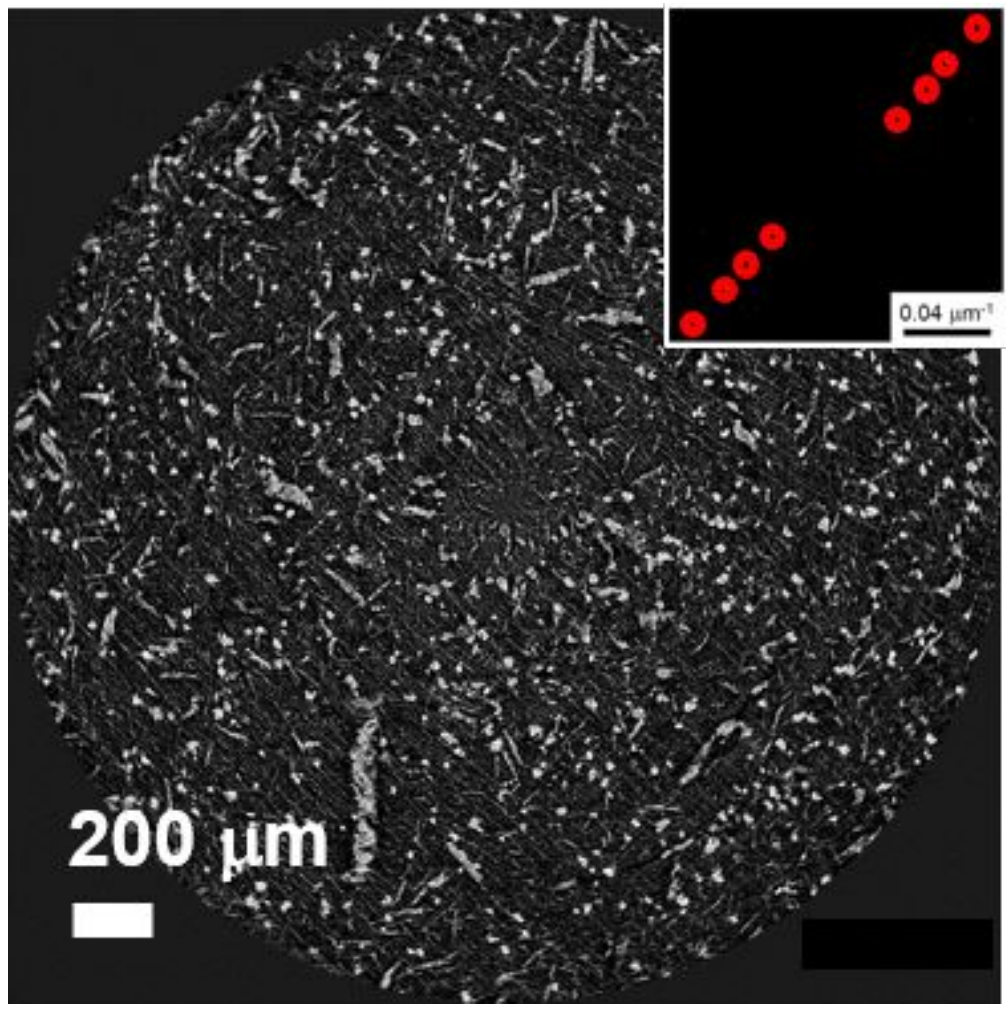

Figure S3 Raw image of the FTC-300 electrode and Fourier transform pattern (inset). The peaks in the FFT pattern are marked by red circles. The interlayer spacing of the FTC-300 electrode is calculated from the FFT pattern.
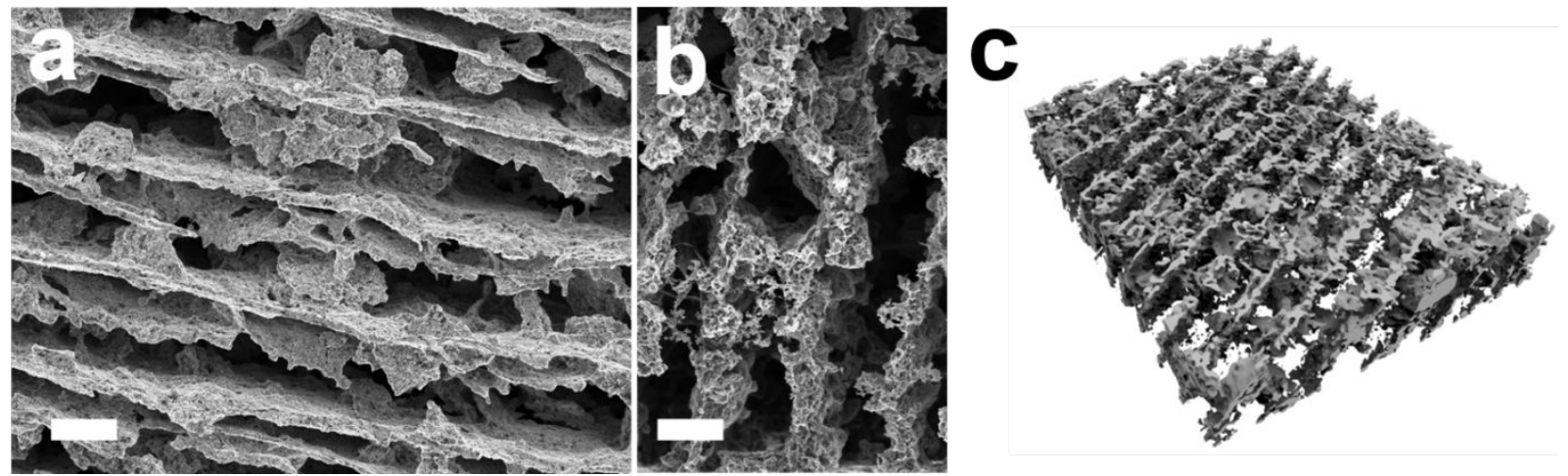

Figure S4 SEM images of the FTC-200 electrode. (a) Top view and (b) cross-section view. (scale bar: $20 \mu \mathrm{m}$ ) (c) Micro CT image of the FTC-200 electrode. (subvolume size: $490 \times 620 \times 120 \mu \mathrm{m}$ ). 


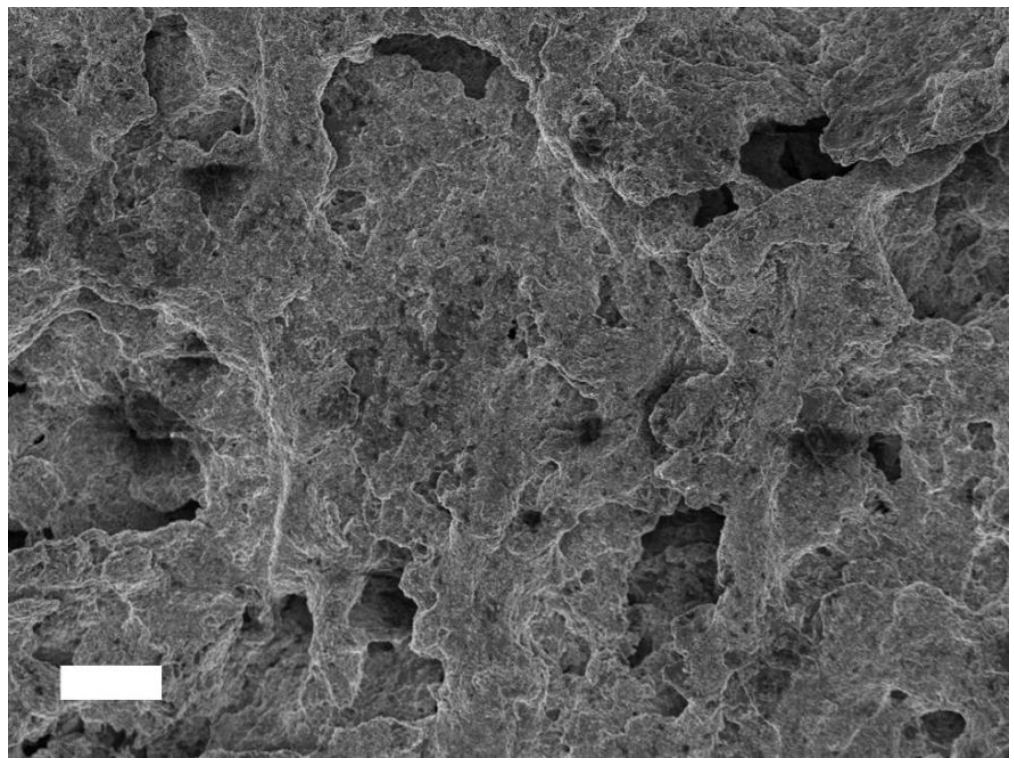

Figure S5 SEM image of the TC electrode (scale bar: $20 \mu \mathrm{m}$ ).

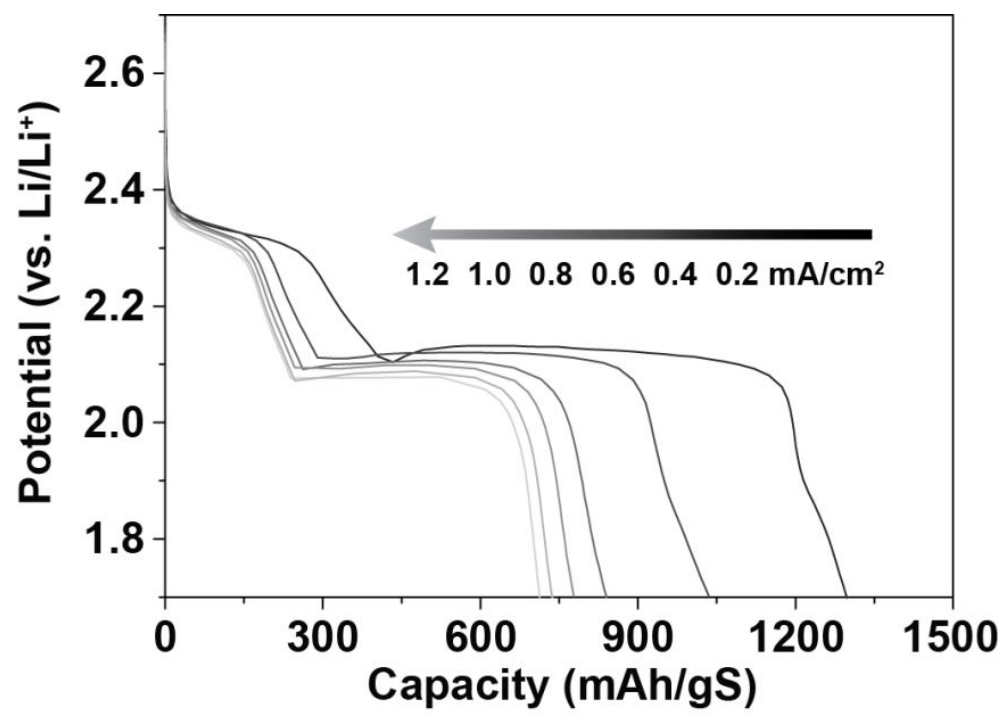

Figure S6 Voltage profiles of the FTC-200 electrode at different current densities. 


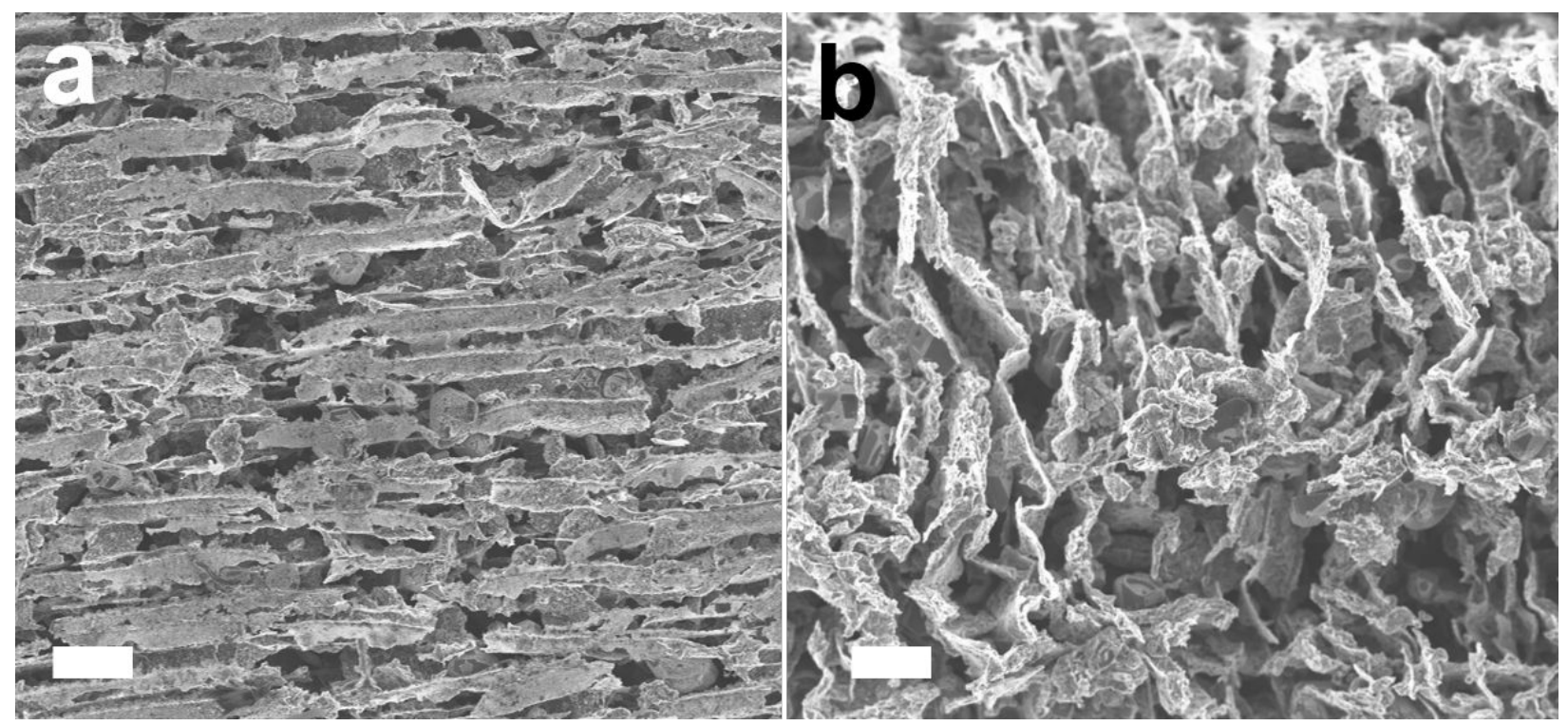

Figure S7 SEM images of the FTC-300 electrode collected from the coin cell. (a) Top view and (b) cross-section view. (scale bar: $20 \mu \mathrm{m}$ )

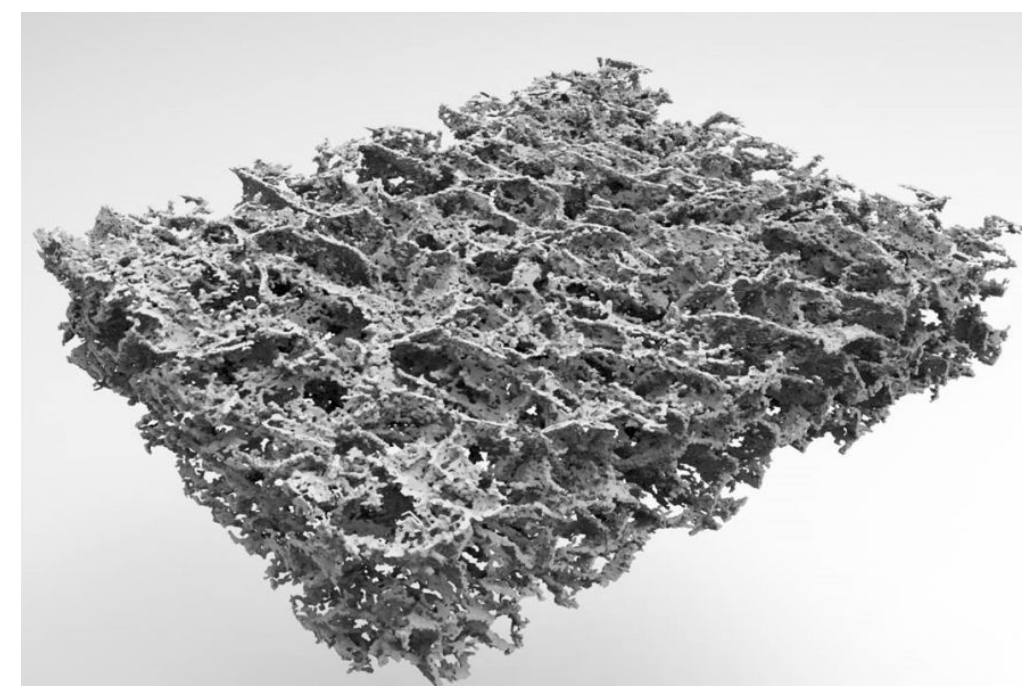

Figure S8 Volume rendering image of the TC-GO electrode (subvolume size: $500 \times 400 \times 100 \mu \mathrm{m}$ ). 
Supporting Information

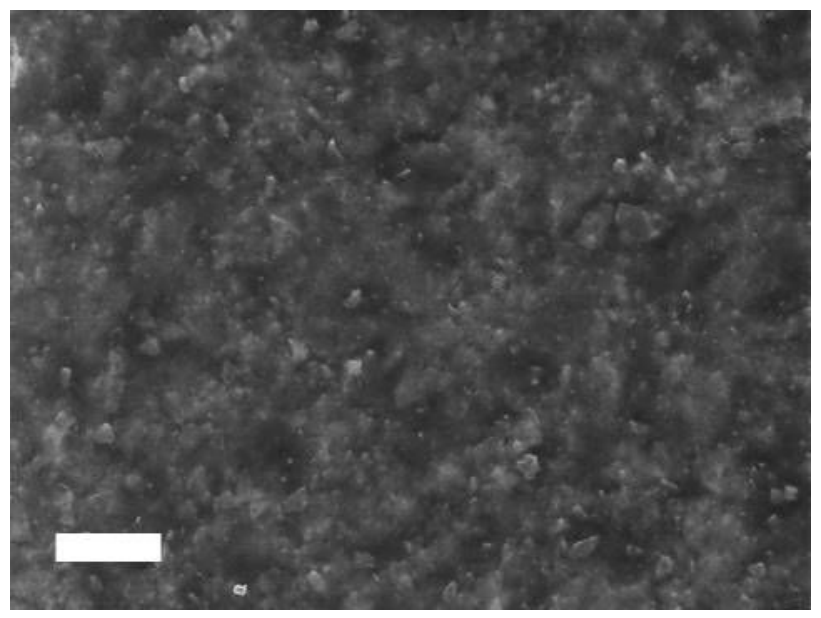

Figure S9 SEM image of a GO film electrode. No porous structure was observed with the particulate binder embedded. (scale bar: $20 \mu \mathrm{m}$ )

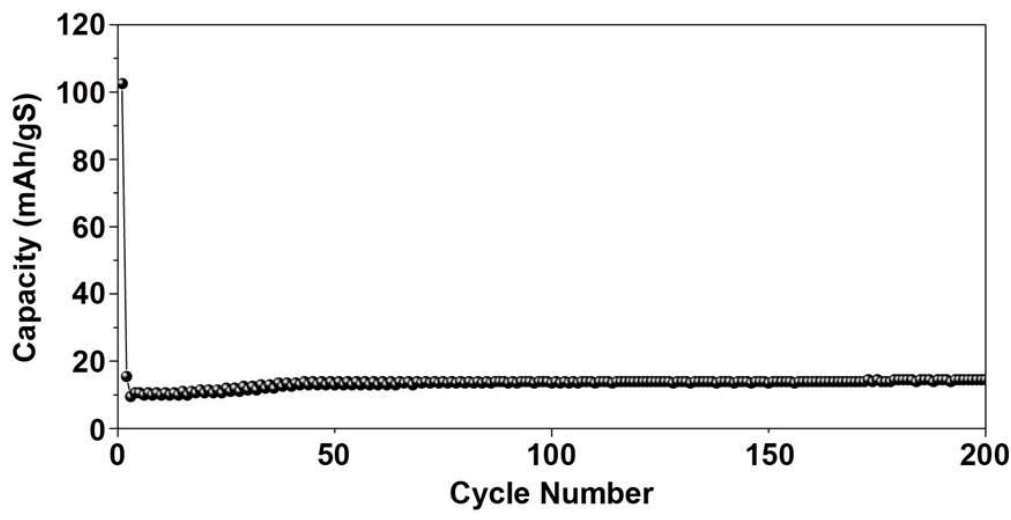

Figure S10 Cycling performance of a GO film electrode at $0.15 \mathrm{~mA} \mathrm{~cm}{ }^{-2}$ for the first cycle and 0.3 $\mathrm{mA} \mathrm{cm}{ }^{-2}$ for the following cycles. The $\mathrm{C}$ rate for the polysulfide catholyte cell was estimated using the weight of sulfur in the catholyte. The polysulfide catholyte is composed of $1 \mathrm{M}$ LiTFSI, $0.1 \mathrm{M}$ $\mathrm{LiNO}_{3}$ and $0.4 \mathrm{M} \mathrm{Li}_{2} \mathrm{~S}_{6}$ in DOL/DME $(1: 1, v / v)$. (Sulfur loading: $\sim 1.8 \mathrm{mgS}$ in catholyte $/ \mathrm{cm}^{2}$ of the GO electrode) 
Supporting Information

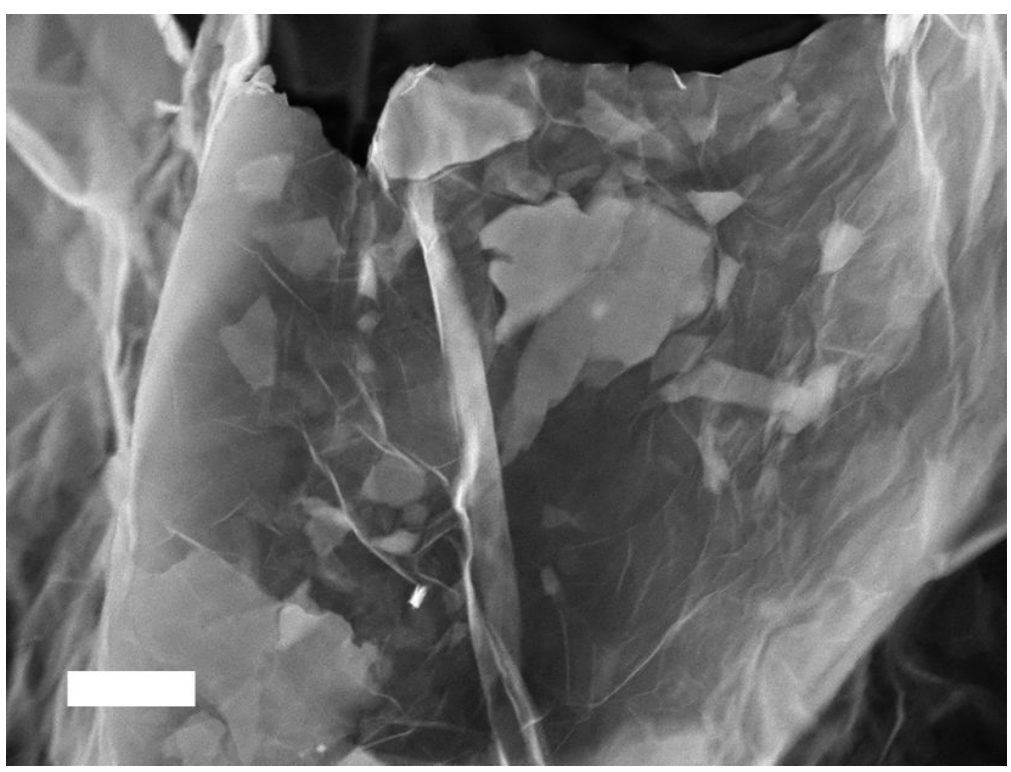

Figure S11 SEM image of the freeze-dried GO powder. (scale bar: $1 \mu \mathrm{m}$ )

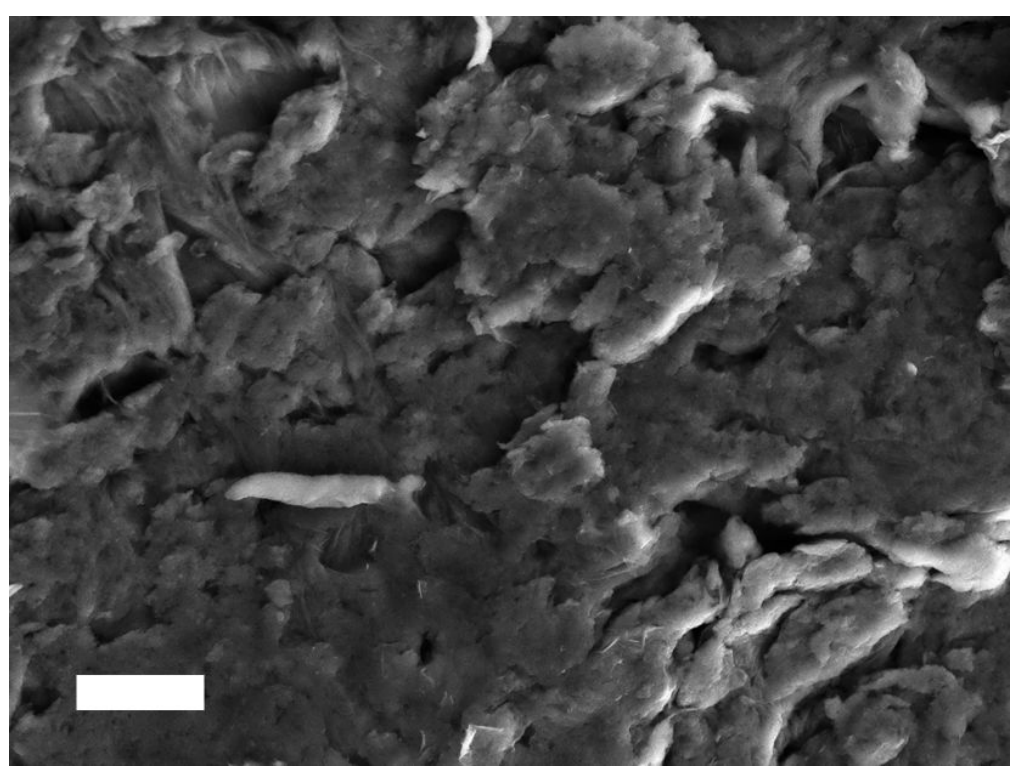

Figure S12 SEM image of the conventionally vacuum-dried GO powder. (scale bar: $1 \mu \mathrm{m}$ ) 


\section{Freeze dried GO}

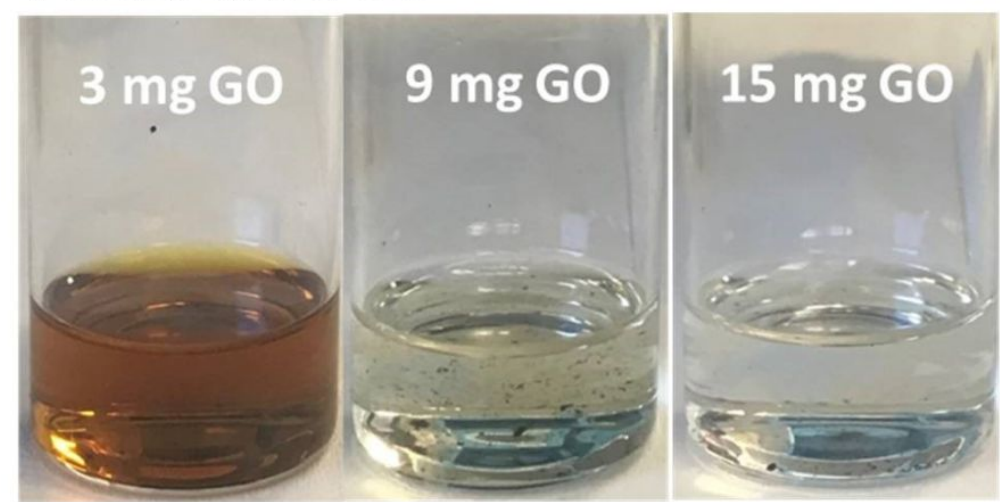

\section{Conventionally dried GO}

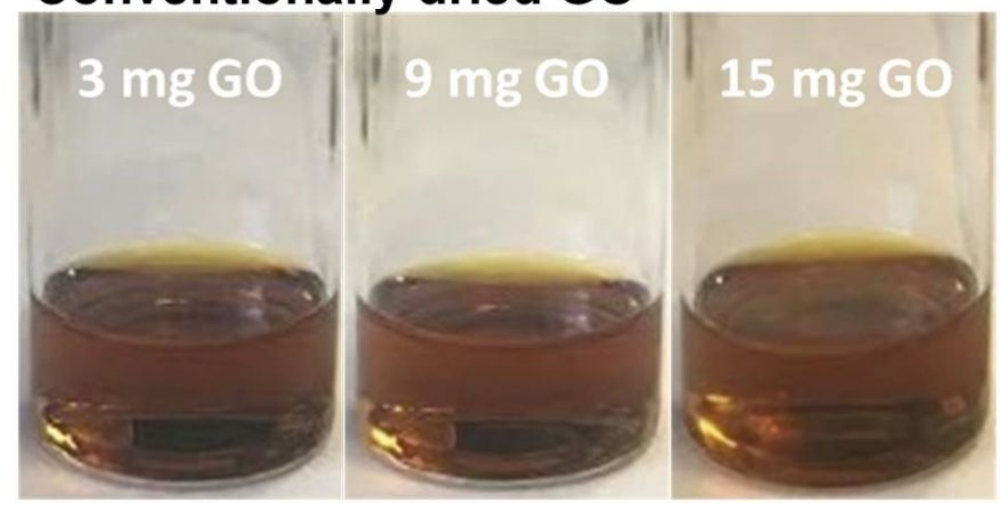

Figure S13 Polysulfide adsorption test results of the freeze-dried GO power and conventionally vacuum-dried GO powder while varying the amount of powder. The polysulfide test solution is composed of $1.25 \mathrm{mM} \mathrm{Li}_{2} \mathrm{~S}_{8}$ in DOL/DME $(1: 1, v / v)$ 


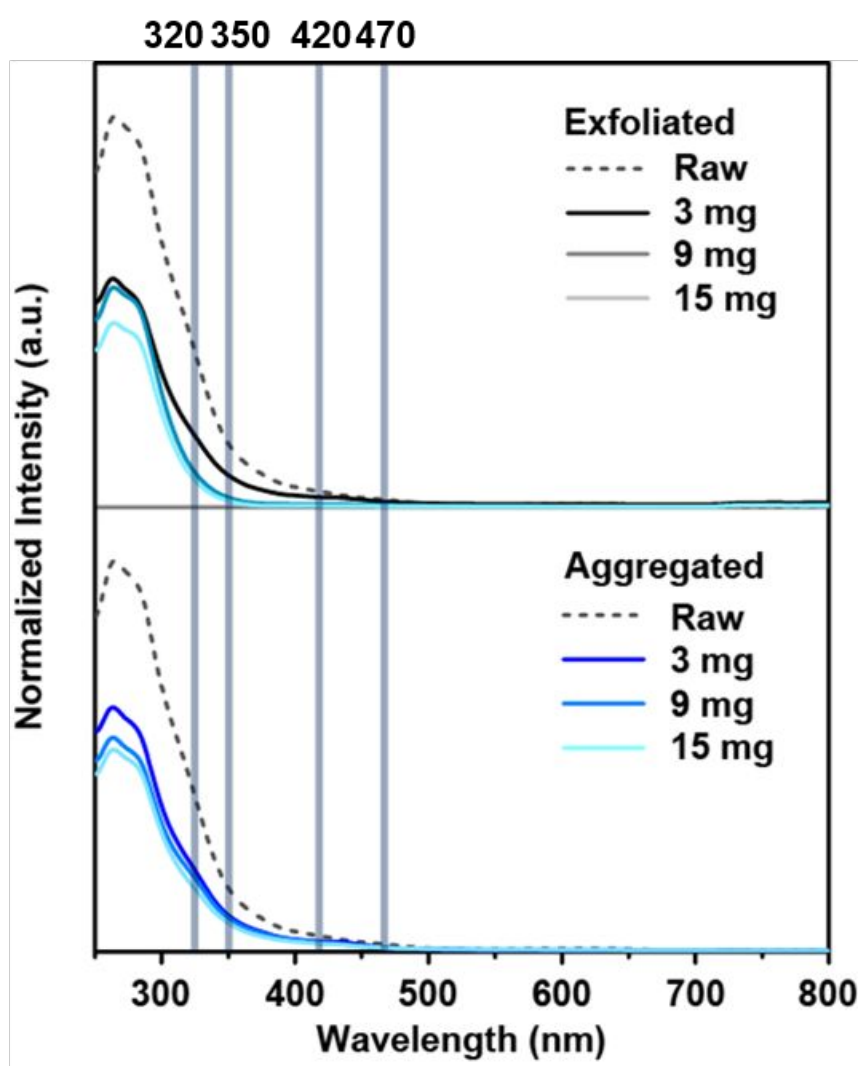

Figure S14 UV-vis test results of the collected polysulfide test solutions. Gray vertical lines indicate the wavelengths for polysulfide species. (Absorbance peaks: $320 \mathrm{~nm},{ }^{3-5} 350 \mathrm{~nm},{ }^{3,5} 420$ $\mathrm{nm}^{3-5}$ and $470 \mathrm{~nm}^{3,5}$ ) Raw indicates the pristine polysulfide test solution without $\mathrm{GO}$ added.

\section{Supplemental References}

1. Song, M.-K., Zhang, Y., and Cairns, E. J., (2013). A Long-Life, High-Rate Lithium/Sulfur Cell: A Multifaceted Approach to Enhancing Cell Performance. Nano Lett., 13, 5891-5899.

2. Hwa, Y., Seo, H. K., Yuk, J.-M. and Cairns, E. J., (2017) Freeze-Dried Sulfur-Graphene OxideCarbon Nanotube Nanocomposite for High Sulfur-Loading Lithium/Sulfur Cells. Nano Lett., 17, 7086-7094.

3. Barchasz, C., Molton, F., Duboc, C., Leprêtre, J.-C. Patoux, S., and Alloin, F., (2012). Lithium/Sulfur Cell Discharge Mechanism: An Original Approach for Intermediate Species Identification. Anal. Chem., 84, 3973-3980.

4. Cañas, N. A., Fronczek, D. N., Wagner, N., Latz, A. and Friedrich, K. A. (2014). Experimental and Theoretical Analysis of Products and Reaction Intermediates of Lithium-Sulfur Batteries. J. Phys. Chem. C, 118, 12106-12114.

5. Zou, Q. and Lu, Y.-C. (2016). Solvent-Dictated Lithium Sulfur Redox Reactions: An Operando UV-vis Spectroscopic Study. J. Phys. Chem. Lett. 7, 1518-1525. 